\title{
ILCEA
}

Revue de l'Institut des langues et cultures

d'Europe, Amérique, Afrique, Asie et Australie

$20 \mid 2014$

Le conte : d'un art à l'autre

\section{Devenir du conte en Union soviétique à la fin des années 1980 : l'exemple des Contes de l'armoise de Jurij Koval'}

The Evolution of Folktales in the USSR in the Late 1980s: Yuri Koval's Polynnye skazki as an Example

СУДЬБА СКАЗКИ В СССР В КОНЦЕ 1980-Х $r$ НА ПРИМЕРЕ ПОЛЫННЫХ СКАЗОК

ЮРИЯ КОВАЛЯ

Laure Thibonnier

\section{(2) OpenEdition}

\section{Journals}

Édition électronique

URL : http://journals.openedition.org/ilcea/2739

DOI : 10.4000/ilcea.2739

ISSN : 2101-0609

Éditeur

UGA Éditions/Université Grenoble Alpes

Édition imprimée

ISBN : 978-2-84310-290-5

ISSN : 1639-6073

Référence électronique

Laure Thibonnier, « Devenir du conte en Union soviétique à la fin des années 1980 : l'exemple des Contes de l'armoise de Jurij Koval' », ILCEA [En ligne], 20 | 2014, mis en ligne le 08 décembre 2014, consulté le 01 mai 2019. URL : http://journals.openedition.org/ilcea/2739; DOI : 10.4000/ilcea.2739

Ce document a été généré automatiquement le 1 mai 2019.

(C) ILCEA 


\title{
Devenir du conte en Union
} soviétique à la fin des années 1980 : l'exemple des Contes de l'armoise de Jurij Koval'

\author{
The Evolution of Folktales in the USSR in the Late 1980s: Yuri Koval's Polynnye \\ skazki as an Example
}

СУДЬБА СКАЗКИ В СССР В КОНЦЕ 1980-Х rr НА ПРИМЕРЕ ПОЛЫННЫХ СКАЗОК

ЮРИЯ КОВАЛЯ

\section{Laure Thibonnier}

Quasiment inconnu en France ${ }^{1}$, Jurij Koval' (1938-1995) est un des écrivains russes de jeunesse les plus célèbres de la seconde moitié $\mathrm{du} \mathrm{xx}^{\mathrm{e}}$ siècle. Dans ses Contes de l'armoise : chronique des temps lointains (Koval', 1987²), il s'inspire des souvenirs d'enfance de sa mère pour ressusciter la Russie rurale du début du xx siècle, " tout le cycle complet de la vie d'un village russe, des semailles à la moisson, de la naissance à la mort » (Koval', 1998).

Le lecteur assiste à la naissance de la petite Lëlja, concomitante à la disparition de son père (2), puis à ses premiers apprentissages : premiers jeux (3), premiers pas (4), premiers mots (4-5). La maman de Lëlja (Tat'jana Dmitrievna) étant institutrice, la fillette grandit dans l'école du village (5-7), appelé Polynovka, car situé dans des steppes envahies par l'armoise (polyn', en russe) ; on en apprend l'histoire (8-10). Lëlja se lie ensuite d'amitié avec Miška, un petit Tsigane égaré dans la steppe et recueilli par l'institutrice (11-14), assiste à la rentrée des classes (15-17), aux premières leçons (18), aux longues soirées passées à se raconter des contes à l'école (19-20), voit la première neige tomber (21-22). Elle découvre bientôt l'injustice: les révolutionnaires enfuis de Sibérie (23-26), la richesse, la pauvreté, l'avarice et la malhonnêteté (27-30), le tout sur fond de courses de luge près de la maison de deux sœurs, qu'on devine moniales et qui incarnent en contraste la bonté et l'amour du prochain (31-33). Avec le printemps, c'est le temps des examens de fin d'études qui arrive (34-37), avant que la maman de Lëlja, malade, n'aille se 
faire soigner à Penza, la ville la plus proche, puis ne revienne auprès de l'enfant, à temps pour voir le lilas fleurir (38-42).

3 Solidement ancrés dans un contexte spatio-temporel précis et centrés autour de la personnalité de Lëlja, les Contes de l'armoise nous renvoient aux questions inévitablement posées par le recours au genre du conte dans la littérature moderne. Quels rapports entre le conte traditionnel oral et la littérature, particulièrement lorsqu'elle s'adresse à la jeunesse ? À quel moment faut-il considérer qu'une œuvre cesse d'être un conte, malgré son titre et la volonté de l'auteur de l'inscrire dans ce genre? La présence d'un fort contexte historique est-elle compatible avec le genre du conte? Ces interrogations se doublent d'un problème plus spécifiquement russe. Utilisé dans les années 1930 par les conteurs officiels pour narrer l'épopée bolchevique, il pourrait apparaître comme un genre discrédité. La démarche de Koval', qui, dans les Contes de l'armoise, recourt à ce genre pour dire l'histoire au moment où l'idéologie soviétique s'épuise, est donc doublement paradoxale, même s'il reste à démontrer qu'on peut encore parler de contes à leur sujet.

\section{Le contexte des contes biographiques du récit-cadre}

De prime abord, les Contes de l'armoise frappent par leur côté presque ethnographique : Koval' y a minutieusement reconstitué la nature, le monde de l'école, et les contextes historique et social de la Russie du début du $\mathrm{xx}^{\mathrm{e}}$ siècle.

\section{I.1. La nature}

La nature est omniprésente dans les Contes de l'armoise. D'une part, les souvenirs de Lëlja sont rythmés par les saisons, leur succession revêtant une telle importance que les contes débutent souvent par l'évocation d'une saison ou d'un phénomène météorologique, parfois reprise dans le paragraphe de conclusion. La fillette fait sa première apparition " par une chaude journée d'été » (p. 8), le livre se clôt sur la floraison du lilas : un cycle de saisons s'est donc écoulé. L'arrivée du printemps donne lieu à un conte entier (35), où l'on voit l'hiver donner littéralement naissance au printemps dans la douleur :

La neige bougeait. Telle un énorme chapeau, elle tressautait et tentait de ramper. Elle n'était pas froide et morte, mais tiède, elle fondait et vivait. Cette nuit, elle s'était sentie mal, comme étouffée et opprimée. Elle se débattait et ne pouvait rien y faire, elle ne pouvait pas se cacher, car elle était énorme.

[...]

Au matin, le grand printemps s'abattit sur le village de Polynovka³. (p. 99)

On voit ici que Koval' fait correspondre les saisons et leurs diverses manifestations (soleil, pluie, neige, tempête...) à des sensations visuelles, mais aussi tactiles, olfactives ou auditives. Ainsi l'été est-il classiquement associé à la couleur de l'herbe, qui finit par imprégner la lumière elle-même, mais aussi au tintement que Lëlja prête à tort aux pissenlits, à leur tiédeur et à leur chatouillement sur ses pieds (p. 8-12). L'automne est doré, entremêlé d'odeurs de poêles, crêpes et gâteaux, mais aussi d'école : craie, encre, tableau noir (p. 45). L'hiver est dominé par la blancheur aveuglante de la neige (p. 59, 62) et les craquements du gel (p. 65); Lëlja use d'associations plus surprenantes lorsqu'elle définit la neige comme duveteuse, tendre et muette (p. 86). En véritable peintre de genre, 
Koval' " voit le verbe littéraire en volume, en couleurs et en musique », ainsi que l'a dit le sculpteur Nikolaj Silis (Bek, 2001).

D'autre part, conformément au mode de vie traditionnel, les saisons rythment également les travaux agricoles et domestiques: les habitants de Polynovka sont décrits comme affairés à la récolte et à la préparation de l'hiver (p.43), confectionnant leurs vêtements et leurs bottes de feutre (p. 43, 81, 25), élevant vaches, moutons et cochons (p. 113). En hiver, on sonne au clocher toutes les demi-heures pour guider les voyageurs égarés dans la steppe; le soir, les élèves se réunissent à l'école où, attablés autour de crêpes, ils écoutent des contes. Dans cette utopie rurale, nature et hommes vivent en harmonie : la nature reflète les sentiments des personnages (le lilas se refuse à fleurir tant que Lëlja est chagrinée par l'absence de sa mère) et les influence :

Les habitants de Polynovka étaient intéressants et très simples. Calmes, façonnés par les steppes et les champs, ils avaient quelque chose de l'armoise. (p. 24)

\section{I.2. L'école}

8 Les souvenirs de Lëlja s'inscrivent également dans le cadre de l'école où enseigne et vit sa mère (3). Aucun détail n'est oublié : la chaire, le tableau avec sa craie et son chiffon, les pupitres, le globe, les rayonnages de livres, les bouliers, les cahiers, les stylos, les plumes et l'encre (6), le compas (23). L'école paroissiale de Polynovka (p. 46) ne comprend qu'une classe unique réunissant trois niveaux de cycle primaire. Tat'jana Dmitrievna fait cours à un groupe, pendant que le deuxième résout des problèmes et que le troisième fait de l'écriture (p.53). Plusieurs chapitres relatent des anecdotes survenues pendant des leçons. Parallèlement aux changements de saisons, deux moments clés sont évoqués : la rentrée, en présence du prêtre et de l'épicier du village, probablement mécène de l'école (17), et l'examen de fin d'études (36-38). Ces deux épisodes créent un autre rythme dans la narration, l'inscrivent dans un temps scolaire différent du cycle saisonnier ; à l'automne, les travaux des champs retardent d'ailleurs la rentrée (p. 43).

En évoquant ainsi les difficultés qu'a rencontrées l'école pour s'imposer dans les campagnes, Koval' inscrit son récit dans un contexte historique précis. Dans la Russie impériale, les écoles paroissiales étaient effectivement courantes dans les campagnes; elles y assuraient un enseignement primaire en trois ou cinq ans. Après l'abolition du servage en 1861, l'enseignement devint universel (1864) et apparurent des écoles accueillant des enfants de toutes les classes sociales. L'amélioration reste, certes, limitée, l'instruction n'étant ni obligatoire, ni forcément gratuite. Mais à la veille de la première guerre mondiale, la Russie tsariste entendait supprimer l'illettrisme d'ici 1920, et nombreux avaient effectivement été les instituteurs envoyés dans les campagnes, comme la mère de Lëlja ${ }^{4}$.

\section{I.3. Le contexte spatio-temporel}

Chaque détail de l'enfance de Lëlja renvoie donc à un contexte spatio-temporel très précis : à travers l'histoire individuelle, se profile l'Histoire. Le cadre géographique des Contes de l'armoise est posé dès le deuxième conte, où l'on voit un cavalier traverser la steppe. Il se précise dans les contes 9 et 11, où est narrée l'origine du village : 
Il y a bien longtemps, dans la nuit des temps, des hommes quittèrent le Nord et ses montagnes escarpées et enneigées pour venir ici. Ils s'arrêtèrent au milieu de la steppe infinie : inondée de soleil, elle les fascinait, et l'odeur de l'armoise les emplissait de joie. Ils s'installèrent dans la steppe, et un village apparut sur la route : Polynovka.

Autour d'eux, il y avait des villages et des villes russes. La terre russe offrit un refuge aux habitants de Polynovka, elle devint leur terre natale.

C'est ainsi qu'un autre peuple, celui de Polynovka, se mit à vivre avec les Russes. Son véritable nom était Mokcha, et cette terre était la Mordovie. (p. 30)

11 Erzya et Mokcha sont les deux groupes constituant le peuple mordve, rattaché à la Moscovie au milieu du XVI ${ }^{e}$ siècle par Ivan le Terrible. Les langues mordves appartiennent à la famille des langues finno-ougriennes, vraisemblablement originaires de l'Oural (les «montagnes du Nord»). À la manière d'un ethnographe, Koval' décrit le costume des jeunes filles de Polynovka (p. 24), et s'intéresse particulièrement à la langue mokcha, "pénétrée du vent de la steppe et d'armoise » (p.30), dont il égrène ici et là quelques mots. Le narrateur ne fait pas de distinction entre russe et mokcha, pas plus que Lëlja qui mélange allègrement les deux langues (11) ou qu'Efimka qui, lors de son examen de fin d'études, remplace dans une poésie l'adjectif « russe » par « de Polynovka », affirmant à l'examinateur qui le reprend que « c'est la même chose » (p. 109).

Malgré cette vision d'une Russie multiculturelle quelque peu idéalisée, la société décrite dans les Contes de l'armoise n'est pas exempte de contradictions et d'injustices. Les contes 28, 30 et 31 opposent, d'un côté, le dévouement de l'institutrice et de la moniale Elenakaj et, de l'autre, la cupidité de l'épicier et du prêtre du village. Derrière les anecdotes rapportées dans ces contes se devinent les tensions de la Russie impériale au début du $\mathrm{xx}^{\mathrm{e}}$ siècle, ébranlée par l'abolition du servage en 1861 et peinant à trouver une place aux roturiers instruits aspirant à des changements sociaux. La révolution de 1917 n'est plus très loin, préfigurée ici par les volčki (" petits loups»), des révolutionnaires enfuis du bagne sibérien que Tat'jana Dmitrievna héberge pour une nuit (26).

Les thèmes de la politique et de la religion sont les moins développés du livre, peut-être parce que Lëlja, comme le lecteur de Koval', est trop petite pour comprendre ce genre de subtilités. Certainement aussi parce que l'écrivain a préféré la prudence en s'aventurant sur le terrain de la politique : les enseignants de l'époque étaient plus souvent socialistes révolutionnaires que bolcheviques, or, les premiers ont été victimes des répressions organisées par les seconds. L'aspect politique n'était d'ailleurs pas forcément le plus intéressant pour un écrivain vivant dans une société fortement idéologisée, d'où peutêtre la tentation de fuir dans le paradis de la nature et de l'enfance.

Quant aux motifs religieux, ils semblent avoir prédominé dans la première version des Contes de l'armoise, mais ont été sabrés par la censure. Selon la définition de Grigorij Oster dans son introduction à un chapitre censuré publié à part ultérieurement, l'œuvre aurait en effet dû être une véritable "encyclopédie de la vie religieuse d'un enfant dans la Russie prérévolutionnaire » (Koval', 1990). Certes, le prêtre apparaît à plusieurs reprises, mais c'est un personnage négatif - non pour des considérations liées à la censure, mais parce que décrit ainsi par la mère de l'auteur (Koval', 1998). En revanche, la foi est évoquée de manière positive, quoique cryptée, à travers Elenakaj et Natakaj, personnages des contes 30 et 32-35, abordant les thèmes de la pauvreté et de la richesse. Désignées uniquement comme des "sœurs", les deux femmes ne sont jamais définies comme des moniales, mais leur description physique et leur comportement ne sauraient tromper un lecteur attentif : 
Grande et maigre, elle [Elenakaj - LT] marmonnait toujours quelque chose, même lorsqu'elle marchait dans la rue. Ses yeux bleus rayonnaient de joie, et elle tendait tendrement les bras à ceux qui venaient à sa rencontre.

- Bonnes gens, disait-elle, aidez-vous les uns les autres, aidez les habitants de Polynovka, tous ceux qui sont en train de geler dans la steppe, qui grandissent, ont faim et souffrent. Quelle étrange vision que cette silhouette noire, bras tendus vers les passants...

Elenakaj prononçait ses paroles tout bas, et tous les passants ne les distinguaient pas. Mais certains tout de même l'entendaient.

Quant à elle, elle voyait, mais n'entendait presque rien. Des personnes comme elle, on dit qu'elles sont « sourdes ». Mais ce n'est pas vrai. Ceux qui sont sourds, ce sont ceux qui ont le cœur sourd. (p. 81)

Même tronqués, les Contes de l'armoise sont bien l'encyclopédie imaginée par Koval'. Leur contexte socio-historique renvoyant au début $\mathrm{du} \mathrm{xx}^{\mathrm{e}}$ siècle russe est facilement identifiable à tout le moins par l'adulte co-lecteur de l'enfant, et semble les distinguer nettement des contes traditionnels pour les inscrire plutôt dans la veine biographique. N'y aurait-il alors entre le conte traditionnel et les Contes de l'armoise qu'une analogie superficielle, résidant principalement dans la facture du texte?

\section{Rapports du récit-cadre et des contes insérés avec le conte traditionnel}

16 Il est difficile de souscrire à cette hypothèse lorsqu'on sait que Koval' avait décidé d'écrire chacun de ses livres dans un genre différent (Koval', 1998) : les Contes de l'armoise sont le fruit d'une réflexion sur le genre du conte et son statut dans le monde de la modernité et de l'écrit. D'autant que Koval' n'avait pas attendu les années 1980 pour s'intéresser à ce genre : son entrée en littérature s'est faite sous le patronage de deux conteurs, Stepan Grigor'evič Pisahov et Boris Viktorovič Šergin, tous deux originaires d'Arkhangelsk, une région fertile en contes (Koval', 1998). Il a même écrit les scénarios de plusieurs dessins animés tirés de leurs contes, pour la plupart justement dans les années 1986-19875. La démarche de Koval' dans les Contes de l'armoise est la même que celle de Šergin, qui a décrit dans ses contes la vie quotidienne des habitants du Nord de la Russie (Šergin, 1936) et l'histoire de la région (Šergin, 1939). Quels parallèles pouvons-nous établir entre les Contes de l'armoise et les contes traditionnels?

\section{II.1. Les contes traditionnels insérés : initiation au contage}

17 Koval' a inséré dans la narration sept contes vraisemblablement traditionnels, que sa mère a effectivement pu entendre dans son enfance : «Conte de Marfuša long comme trois crêpes ${ }^{6}$ » (10), «Conte du frère de la steppe, raconté par Marfuša » (15), «Conte du grand-père Ignatij et du loup Evstifejko ${ }^{7}$ » (21), "Conte de trois autres roubles, raconté par grand-père Ignatij » (29), «Conte du faucon d'argent, raconté par Natakaj » (33), «Conte du sorbier, raconté par Dunja » (40) et «Conte du bouc Koz'ma Mikitič, raconté par grand-père Ignatij » $\left(42^{8}\right)$.

Certains de ces contes mettent en scène des animaux : le conte 10 est un conte-chaîne ; dans le conte 21 , le grand-père Ignatij triomphe d'un loup trop glouton et, dans le conte 42 , c'est un bouc qui se joue des loups. Le conte 29 relève de la catégorie des contes 
anecdotiques. On trouve également des contes merveilleux : dans le conte 15, on relèvera un frère disparu et des adversaires surnaturels (Virjava, un démon), dans le conte 33 , une jeune fille transformée en bouleau qu'épousera le héros.

Ces contes puisent aussi bien dans le répertoire slave que finno-ougrien. Pour l'apport slave, on rapprochera le conte 33 du conte $n^{\circ} 275$ d'Afanas'ev, «Le bouleau et les trois faucons » (Afanas'ev, 1984-1985, t. 2, p. 2839), et le conte $42 \mathrm{du}^{\circ}$ 554, «Conte du bouc et du mouton" (Afanas'ev, 1984-1985, t. 3, p. 22010). Du côté finno-ougrien, on trouve Virjava, l'équivalent mordve de la Baba-Yaga russe. Dans la série de dessins animés $L a$ Montagne de pierres fines [Gora samocvetov ${ }^{11}$, le conte 42, recueilli par Afanas'ev en ukrainien, est présenté comme un conte carélien, et donc finno-ougrien. Cette migration du sujet, du groupe linguistique slave au groupe finno-ougrien, fait écho à la situation de Lëlja, qui connaît le russe et le mokcha.

Ces contes sont des récits enchâssés, toujours introduits par la description d'une scène de contage et rattachés à une figure de conteur précise, mentionnée jusque dans les titres. Marfuša est la jeune écolière, bavarde et joyeuse, qui rapporte un conte d'enfant (le conte-chaîne) et le conte merveilleux du frère de la steppe. Le conte merveilleux du faucon argenté est raconté par Natakaj ; celui de Dunja narre l'amour et la tristesse d'un sorbier ${ }^{12}$ et d'un chêne séparés par une rivière (on relèvera la réutilisation du sujet du poème d'Ivan Surikov, Čto šumiš, kačajas' / Tonkaja rjabina..., dont la variante chantée est très populaire). Toutes trois sont des figures maternelles, qui prennent soin de Lëlja en l'absence de sa mère. Ainsi se dessine le profil d'une conteuse spécialisée dans les contes pour enfants ou merveilleux, traitant d'amitié ou d'amour. Grand-père Ignatij, lui, seul conteur masculin, est un personnage bourru, dont la phrase la plus longue semble d'ordinaire être « Nous voilà bien rendus! » («Vot i priehali !», p. 22-23). Mais, pour peu qu'on insiste, de ses grommellements naît bientôt un conte qui, cette fois, sera plus effrayant (p. 55). Derrière ces figures se profile un autre conteur, moderne : l'auteur luimême, qui adopte tantôt la position du narrateur classique (dans le premier conte, il se représente malade, se remémorant les contes que lui racontait sa mère dans son enfance), tantôt celle d'héritier de Marfuša et Ignatij :

Ses contes, Marfuša les a racontés il y a bien longtemps, et bien des années ont passé avant que je les entende, et bien d'autres années ont passé avant que je ne vous les raconte. Aujourd'hui, Marfuša a quitté ce monde, mais moi, je suis toujours vivant. Écoutez donc le conte de Marfuša tel que je vous le raconterai. (p. 25)

De l'eau a coulé sous les ponts depuis, et grand-père Ignatij n'est plus de ce monde. Mais moi, je suis toujours là. Alors écoutez le conte de grand-père Ignatij tel que je vous le raconterai. (p. 55)

21 Dans ces formules introductives, Koval' rappelle une caractéristique fondamentale du conte, venu du fond des temps et transformé par chaque conteur, pour enjoliver le récit. Plus loin, l'écrivain distingue, pour un même conte, une différence entre la façon dont le conteur le dit, et celle dont son auditoire le retiendra et le colportera ensuite à son tour : « Dunja chantait, et Lëlja écoutait, et voici comment Lëlja a retenu ce conte » (p. 110). Il effleure ici la notion d'élaboration secondaire, que le rêve et le conte partageraient, selon Nicole Belmont : pour cette dernière, les conteurs «proposent des images mentales aux auditeurs », que ceux-ci « reçoivent, [...] décryptent et [...] élaborent au plus profond de leur inconscient» (Belmont, 1999: 230). Ces formules alliées aux descriptions de scènes de contage, donnent le sentiment que Koval' a conçu ses Contes de l'armoise comme une initiation à l'art perdu du contage. 


\section{II.2. Les contes « biographiques » : la réalité vue par les yeux du conte} Lëlja) les procédés du conte traditionnel. Ici, les parallèles sont nombreux, à commencer par la langue de Koval', bigarrée, imagée et comique à souhait. On retrouve dans ces contes les particularités linguistiques communes aux contes russes, par exemple les épithètes récurrentes (Miška, le « soldat errant ») ou les diminutifs (dans le conte 33, la jeune fille transformée en bouleau est parfois appelée berëzka, "petit bouleau»). Les idiotismes sont renforcés par le recours fréquent aux dialogues, qui permettent d'insérer des tournures plus orales. Ce procédé culmine dans les contes insérés, par exemple dans cette réplique du soldat dans le conte 33 :

- Bon, dit le soldat, compris, pas compris, ce n'est pas ton affaire. Je n'ai pas le temps de palabrer avec vous, les bouleaux. Reste bien là, dans la terre, et attends, au lieu de jacasser avec le premier soldat venu. (p. 91)

Le personnage du prêtre semble d'ailleurs sorti tout droit d'un conte: sa grande taille, son vêtement noir, sa coiffe ressemblant à une cheminée qui laisserait échapper sa fumée par le bas (la barbe) (p. 20-21) font penser à un dragon. Sa grosse voix et les prières en slavon d'Église, incompréhensibles pour les Mordves, évoquent un magicien prononçant des formules magiques; c'est d'ailleurs ainsi que finissent par le voir les habitants de Polynovka, lorsqu'ils entendent son gramophone, autre nouveauté dans cette contrée (p. 83-84). candidats à l'examen de fin d'études, puis des examinatrices, et enfin du curateur (p. 104-106) reprend le principe de la répétition ternaire. La procession des quatre candidats, endimanchés, suivis de leurs parents chargés de présents, devant le village rassemblé au pied de l'école (p. 104), fait penser au départ d'adolescents prêts à subir un rite d'initiation. Les examinatrices et le curateur ressemblent à des divinités menaçantes, mais, comme dans le conte, les passages effrayants sont entrecoupés d'éléments comiques comme les remarques de Kuzja, cousin de l'idiot du conte traditionnel, qui désacralisent l'atmosphère de rituel de l'épisode ("À coup sûr, elles sont plus importantes qu'un général [...]. Mais ça existe, des bonnes femmes plus importantes qu'un général?", p.106), ou la description physique du curateur, porteur de tous les signes de l'élite sociale : un chapeau, des lunettes et, surtout, un nez, des moustaches et des joues bien roses (p. 106). À peine descendu de son équipage, ce personnage hors du commun est confronté au prêtre du village, bien décidé à sauvegarder son statut de grand mage, dans un affrontement humoristique typique du conte :

À peine le Curateur fut-il descendu de voiture que le prêtre fit son apparition. Cela faisait longtemps qu'il guettait à la fenêtre l'arrivée de monsieur le Curateur. Se montrer plus tôt aurait été gênant.

Le Curateur vint à sa rencontre, le prêtre le bénit, et monsieur le Curateur embrassa sa main. À cet instant, aux yeux des villageois, le prêtre réintégra les cieux dont il était quelque peu descendu dans les instants qui avaient précédé. (p. 106-107)

Certains éléments constitutifs du conte traditionnel migrent vers les passages biographiques, et, à son tour, la réalité pénètre dans le conte. Ainsi, contrairement à la plupart des contes traditionnels, les contes insérés des Contes de l'armoise sont localisés : à 
l'exception des contes 33, 42 et éventuellement $10^{13}$, ils ont pour cadre Polynovka ou ses environs. Par ce manquement aux règles tacites du conte, Koval' brouille la frontière entre réalité et conte, et rend à ce dernier une actualité étonnante : le conte cesse ici d'être un héritage du passé pour devenir un genre vivant, susceptible de livrer un témoignage sur une époque historique récente, à savoir la fin de la Russie impériale.

\section{II.3. Conte, histoire et enfance}

Or, paradoxalement, Koval' confond dans un même texte histoire, conte et mythe, par exemple dans le second conte, qui fait office de récit originel: on y voit un cavalier traversant la steppe de nuit pour rejoindre sa femme et son enfant sur le point de naître - la petite Lëlja verra le jour, mais une chute mettra fin aux jours du cavalier (son père). Ce tableau cosmogonique est dominé par les noirs et les gris de la nuit, des nuages et des pierres, sur lesquels tranchent le blanc de la foudre et le rouge du feu des bergers. On y trouve trois des quatre éléments (le feu, la terre et l'air), mais aussi les figures bibliques de l'agneau et du pasteur. Dans ce chaos primordial, la steppe se confond avec le ciel, l'inanimé devient vivant (les pierres se trouvent être des moutons), et, en mourant, le masculin (le cavalier) donne naissance au féminin (Lëlja) (on songera à Athéna sortant du crâne de Zeus).

Or, ce conte n'est pas seulement le récit des origines de Lëlja, mais aussi celui de la naissance de la Russie soviétique: à la veille de la disparition de l'URSS née en octobre 1917, Koval' rappelle la préhistoire de cette société, ancre son époque dans la Russie impériale et, au-delà, traditionnelle. Cette visée historique est d'ailleurs soulignée par le sous-titre des Contes de l'armoise - Chronique des temps lointains -, qui rappelle la Chronique des temps passés (Povest' vremennyh let, v. 1113), la plus ancienne compilation historique de la Russie ancienne parvenue jusqu'à nous. La Chronique des temps passés renvoie à une époque tellement éloignée de nous qu'elle peut aujourd'hui sembler se perdre dans les limbes de l'Histoire. Les événements qu'elle rapporte sont certes répartis chronologiquement, année par année. Mais le compte du temps se faisant à partir de la création du monde, le lecteur $\mathrm{du} \mathrm{xx}^{\mathrm{e}}$ siècle est rapidement désorienté (ainsi la première date mentionnée est l'année 6360, qui correspond à l'an 852). Les Contes de l'armoise, eux, renvoient à une période bien plus récente, que le lecteur russe n'aurait aucun mal à dater, bien qu'aucune date n'y soit mentionnée. Pourtant, le voisinage entre les contes traditionnels enchâssés et les épisodes biographiques, présentés sous l'apparence de contes, tend à brouiller les repères chronologiques, à faire entrer l'enfance de Lëlja dans un cadre intemporel. Peut-être Koval' a-t-il souhaité ainsi insister sur la rupture entre la Russie impériale et l'URSS ? Ou bien, au contraire, a-t-il voulu, en faisant appel au conte et au mythe, qui supposent tous deux l'existence d'une vérité cachée, donner une expression imagée d'un tabou, ici historique : celui d'une continuité entre les périodes impériale et communiste, alors même que la société soviétique était proclamée nouvelle ${ }^{14}$ ?

Dans le même temps, les Contes de l'armoise narrent l'histoire individuelle de Lëlja. La narration est souvent menée du point de vue de l'enfant (voir le portrait d'Ignatij dans le conte 8). Les premiers contes sont consacrés à l'apprentissage de la langue par Lëlja : elle pense, mais ne sait pas exprimer ses pensées par la parole (3). Ensuite, ses jeux avec la langue donnent naissance à des contes: les contes 5,6 et 8 sont basés sur l'étymologie commune entre krylo (l'aile), et kryl'co (le perron). Avec l'âge, les histoires inventées par 
Lëlja, pour s'expliquer le monde, se mettent à ressembler à de véritables contes (28). Par ailleurs, les Contes de l'armoise répondent à un impératif du conte, en mettant en scène les étapes à franchir par l'enfant pour se séparer de sa mère, son initiation à une vie autonome : apprentissage de la marche, du langage, puis première séparation de Lëlja avec sa mère, partie se faire soigner en ville. Ainsi, en identifiant conte et regard de l'enfant sur le monde, Koval' abolit la frontière qu'on fait trop souvent passer, de nos jours, entre le conte (forcément fantaisiste) et la réalité (véridique). Le narrateur adopte le regard faussement naïf de l'enfant pour se donner la possibilité d'assouplir les règles de la vraisemblance historique. Par le biais du regard de l'enfant, Koval' réussit à la fois à concilier le genre du conte et le genre biographique, mais aussi à rendre au conte sa propension à livrer un témoignage historique. Ainsi le lecteur des Contes de l'armoise est-il invité à une triple initiation : à l'art de dire et d'écouter un conte, à celui de grandir, et à celui, encore plus ardu, de jeter un regard neuf sur l'histoire de la Russie après plusieurs décennies de doxa soviétique.

\section{BIBLIOGRAPHIE}

AFANAS'EV Aleksandr (1984-1985), Narodnye russkie skazki A.N. Afanas'eva, $v 3 t$. [Contes populaires russes d'A. N. Afanas'ev, en 3 tomes], Moscou : Nauka.

BEK Tat'jana (2001), « Naiosobyj opyt ogromnoj sily » [« L'expérience singulière d'une force considérable »], Literatura [Littérature], 15, Moscou : izd. dom « Pervoe sentjabrja », <http:// lit.1september.ru/2001/15/lit15_05.htm> (24 octobre 2011).

BERELOWITCH wladimir (1990), La Soviétisation de l'école russe, Lausanne : L'Âge d'Homme.

KOVAL' Jurij (1987), Polynnye skazki: Povest' o davnih vremenah [Les contes de l'armoise: Chronique des temps lointains], Moscou : Detskaja literatura.

KOVAL' Jurij (1990), «Skazka o kolokol'nyh brat'jah » [ «e conte des frères du clocher »], Ogonëk [ Petite flamme], 34, <http://community.livejournal.com/suer_vyer_/122716.html> (23 octobre 2011).

KOVAL' Jurij (1998), « Ja vsegda vypadal iz obščej strui ». Besedu vela I. Skuridina [«J'ai toujours nagé à contre-courant ». Discussion avec I. Skuridina], Voprosy literatury [Questions de littérature], 6, <magazines.russ.ru/voplit/1998/6/kov.html> (23 octobre 2011).

KOVAL Youri (1977), Le Renard bleu s'est échappé, Paris : Hachette.

ŠERGIN Boris (1936), Arhangel'skie novelly [Nouvelles d'Arkhangelsk], Moscou : Sovetskij pisatel'. ŠERGIN Boris (1939), U pesennyh rek [Près des rivières de chants], Moscou : Hudožestvennaja literatura. 


\section{NOTES}

1. Le lecteur curieux ne trouvera en français qu'une seule traduction : celle de Nedopësok, publiée sous le titre Le renard bleu s'est échappé et effectuée à partir de l'allemand et non de l'original russe (Koval, 1977). Le film tiré de cette œuvre en 1978 est également sorti en France, sous le titre Napoléon III le petit renard bleu (Socai films, 1986).

2. Sauf mention contraire, les citations seront issues de cette édition; les numéros de page, précédés de la mention « p. », seront indiqués entre parenthèses, sans référence à l'édition. Par ailleurs, Koval' n'a pas numéroté ses contes, mais, par souci de concision, nous avons pris la liberté de le faire ; les numéros entre parenthèses renvoient donc aux contes.

3. En l'absence de traduction française, toutes les citations des Contes de l'armoise seront traduites par nos soins.

4. Sur l'école russe au début du Xx siècle, voir Berelowitch, 1990.

5. Il s'agit des dessins animés Nouvelles d'Arkhangelsk [Arhangel'skie novelly, 1986], inspiré de Pisahov, L'anneau magique [Volšebnoe kol'co, 1979], Véritables histoires pomores [Pomorskaja byl', 1987], La pluie [Dožd', 1991], Mister Pron'ka (1991) d'après Šergin, et de Rire et malheur sur la mer Blanche [Smeh i gore u Bela morja, 1987], qui emprunte aux deux conteurs.

6. Associé à des motifs issus des contes 2 et 3, il a été porté à l'écran en 2003 par G. Šakickaja sous le titre Conte de Polynovka long comme trois crêpes [Polynnaja skazka $v$ tri blina dlinoj].

7. Il existe également un dessin animé reprenant ce conte, Le loup Evstifejka [Evstifejka-volk, 2001] de E. Užinova et O. Užinov.

8. Là encore, ce conte a inspiré les réalisateurs de dessins animés, puisqu'il en existe deux versions, celle de E. Berezovaja et A. Tatarskij en 2004 (Sur le mouton et le bouc [Pro barana i kozla]) et celle de S. Glagolev en 2005 (Sur le bouc et le mouton [Pro kozla $i$ barana]).

9. Ce conte se trouve dans le tome 3 de la traduction française des contes d'Afanassiev par L. Gruel-Apert, sous le $\mathrm{n}^{\circ} 213$ (Afanassiev, 2009-2010).

10. Dans la traduction de L. Gruel-Apert, ce conte figure dans le tome 1, sous le numéro 30, et porte le titre «L'ours et les loups effrayés » (Afanassiev, 2009-2010). Il s'agit de la traduction du conte 44/19a d'Afanassiev en russe.

11. Voir la note 8 .

12. En russe, rjabina (le sorbier) est de genre féminin.

13. Ce dernier conte n'est pas expressément situé à Polynovka, mais il est facile de faire le lien entre l'appétit de la souris pour les crêpes et celui des villageois.

14. Même si cette idée peut sembler banale aujourd'hui, les réticences de la censure à l'égard des Contes de l'armoise évoquées ci-dessus montrent bien que cette interprétation n'allait pas de soi.

\section{RÉSUMÉS}

Le présent article est consacré aux Contes de l'armoise [Polynnye skazki] de Jurij' Koval', basés sur les souvenirs d'enfance de la mère de l'écrivain. Nous nous arrêtons sur deux aspects apparemment contradictoires de ce texte : le réalisme du contexte et le merveilleux du conte. Nous montrons comment certains éléments des contes populaires insérés dans la narration 
influencent le récit de l'enfance de Lëlja, à son tour exposée dans le contexte historique plus large du début $\mathrm{du} \mathrm{xx}^{\mathrm{e}}$ siècle russe. Le conte devient ainsi le lieu de rencontre entre les histoires collective et individuelle.

This paper studies The Folktales of mugwort [Polynnye skazki] by Yuri Koval', based on his mother's childhood memories. We will focuse on two apparently contradictory aspects of this text: the realism of the context and the wonderful aspect of folktales. We will show how some elements of the bona fide folktales present in the narration influence the way Lëlja's childhood is shown. In the same time, this individual story is a part of the larger context of the early 20th century. Therefore, the folktale becomes the place where collective history and individual story meet.

ДАННАЯ СТАТЬЯ ПОСВЯЩЕНА ПОЛЫННЫМ СКАЗКАМ ЮРИЯ КОВАЛЯ, ОСНОВАННЫМ НА ДЕТСКИХ ВОСПОМИНАНИЯХ МАТЕРИ ПИСАТЕЛЯ. В НЕЙ РАССМАТРИВАЮТСЯ ДВА НА ПЕРВЫЙ ВЗГЛЯД ПРОТИВОПОЛОЖНЫХ АСПЕКТА ЭТОГО ТЕКСТА - РЕАЛИЗМ В ОПИСАНИИ КОНТЕКСТА И ВЛИЯНИЕ СКАЗКИ. ПОКАЗЫВАЕТСЯ, КАК НЕКОТОРЫЕ ЭЛЕМЕНТЫ НАРОДНЫХ СКАЗОК ВЛИЯЮТ НА РАССКАЗ О ДЕТСТВЕ ЛЁЛИ, КОТОРОЕ В СВОЮ ОЧЕРЕДЬ ВКЛЮЧЕНО В БОЛЕЕ ШИРОКИЙ ИСТОРИЧЕСКИЙ КОНТЕКСТ НАЧАЛА ХХ-ГО ВЕКА В РОССИИ. ТАКИМ ОБРАЗОМ СКАЗКА СТАНОВИТСЯ МЕСТОМ, ГДЕ ВСТРЕЧАЮТСЯ КОЛЛЕКТИВНАЯ И ИНДИВИДУАЛЬНАЯ ИСТОРИИ.

\section{INDEX}

motsclesru КОВАЛЬ, ПОЛЫННЫЕ СКАЗКИ, РУССКАЯ ДЕТСКАЯ И ЮНОШЕСКАЯ ЛИТЕРАТУРА, СКАЗКА, ИСТОРИЯ

Keywords : Koval, Polynnye skazki, russian children's and juvenile literature, folktale, history

Mots-clés : Koval, Contes de l'armoise, littérature d'enfance et de jeunesse russe, conte, histoire

\section{AUTEUR}

\section{LAURE THIBONNIER}

Université Grenoble Alpes, ILCEA 